\title{
Crop Yield Skewness Under Law of THE Minimum TeChNOLOGY
}

\author{
David A. Hennessy
}

\begin{abstract}
No satisfactory motivation has been forwarded in favor of any crop yield distribution, including the normal. This article explores the foundations of yield distributions for the Law of the Minimum resource constraint technology at the plot level of analysis. With independent, identical, uniform resource availability distributions the yield skew is positive, whereas it is negative whenever the distributions are normal. Simulations show how asymmetries in resource availabilities determine skewness. It is suggested that a negative yield skew occurs whenever production is tightly controlled so that the left tails of some resources availabilities distributions are thin. Irrigation may increase yield skewness.
\end{abstract}

Key words: beta-normal distribution, crop insurance, Liebig technology, limiting factors, order statistics, reliability, weakest-link.

Two major and unresolved themes in the production economics of crop agriculture concern responses to inputs absent uncertainty and yield distributions conditional on inputs. Nature, through sunshine, rainfall, and other weather variables, ensures that crop inputs are stochastic. In addition, inputs applied during cultivation do not equate with inputs available to the plant, in part because of weatherdependent linkages involving soil attributes. Thus, these two themes cannot be separated. If little is known on input-output relations in the absence of uncertainty about input availabilities then little can be known on these relations in the presence of uncertainty. Using a microfoundations approach, this article explores how the deterministic structure of crop production can affect yield distributions at the small-plot level of analysis. In doing so, we pay particular attention to one controversial feature of yield distributions, crop yield skewness.

To further these goals, a stance must be taken on the deterministic structure of crop production technologies. Although long controversial, the only technology with clearly motivated foundations is the Sprengel and von Liebig "Law of the Minimum," or the LoM for short. The idea is that crop input availabilities are perfect complements such that the most limiting determines output; for example, $y=\min \left[\varepsilon_{1}\left(x_{1}\right), \varepsilon_{2}\left(x_{2}\right), \ldots\right]$ where resource

David A. Hennessy is professor at the Department of Economics and affiliate of the Center for Agricultural and Rural Development (CARD), Iowa State University.

Detailed and helpful comments from two anonymous referees and editor Walter N. Thurman are gratefully acknowledged. availability (or RA) $\varepsilon_{i}\left(x_{i}\right)$ is a nondecreasing function of some input vector $x_{i}$. When the $\varepsilon_{i}\left(x_{i}\right)$ are linear functions then the LoM technology is said to be linear. In general, the technology requires that surplus RAs have null marginal product. ${ }^{1}$ When advocating the technology, von Liebig famously suggested the analogy with what is now referred to as the Liebig barrel. This barrel has a regular bottom but stave lengths differ at the top. Capacity is determined by the shortest stave so that lengthening any other staves has no effect. The claim has found some, but limited, empirical support.

Paris (1992) used Iowa corn production experiment data to find support for a nonlinear LoM specification, where Frank, Beattie, and Embleton (1990) had found evidence against a linear version with that data set. Using nonparametric data envelopment methods on Iowa corn data, Chambers and Lichtenberg (1996) identified mixed results on input substitution implications for the specification. Berck, Geoghegan, and Stohs (2000) took a nonparametric regression approach to test for the absence of input substitution and found little support for the LoM.

In the agronomy literature, Cerrato and Blackmer (1990) are among many who have favored the form. Others, as in Bloom, Chapin, and Mooney (1985), point to a multiple limitation hypothesis or MLH. The MLH uses

\footnotetext{
${ }^{1}$ The weakest-link technology also arises in the economics of financing public goods (Hirshleifer 1983; Cornes 1993), as well as in health economics (Dow, Philipson, and Sala-I-Martin 1999).
} 
an economic framework, viewing nutrients resources to be allocated within the plant to conclude that "Growth is equally limited by all resources," (Bloom, Chapin, and Mooney 1985, p. 367). Emphasizing evolution, this literature posits that successful species evolve to support biological pathways that best substitute for limiting resources when at risk. For example, one means of effecting substitution is to store nutrients (at a cost) for possible later use. Laboratory tests, as in Rubio, Zhu, and Lynch (2003), of these alternative hypotheses on a wide range of nutrients have not been decisive. The LoM appears to be appropriate for many nutrient comparisons while for others the production process is more involved.

Thus, research is quite inconclusive on the LoM. A possible reason for this, at least with some data sets considered above, is the role of spatial nonuniformities. Berck and Helfand (1990) have pointed out that integration over such nonuniformities can smooth away nondifferentiable points in a LoM technology so that the observed noisy data may rationalize an alternative technology. Our modeling framework will assume a generalized LoM technology in the presence of noise in order to understand crop yield distributions.

The literature on yield distributions, though not as extensive, is also unresolved. As with identifying the nature of a deterministic technology, the complexity of a biological system requires careful conditioning of the environment to test for technical attributes. Even under experimental conditions, field cropping is far from ideal in this regard. Parallel to the LoM, there also exists a yield distribution that is advocated by reference to theoretical foundations, the normal. Here, the idea in the background is often that yield realizations over a sufficiently large area will differ due to many distinct shocks. The reasoning goes that some central limit theorem (CLT) can be invoked to identify the normal, with zero skewness, as the limiting distribution.

The most widely cited early work on yield distributions is by Day (1965). His data were from nitrogen-conditioned experimental cotton, corn, and oats plots in Mississippi over the middle twentieth century. While finding strong evidence for positive skewness (loosely, where probability mass tapers off less quickly to the left of the mean than to the right) in cotton, there was weaker evidence for positively skewed corn yields and fairly strong evidence for negatively skewed oats yields. In addition, his skewness estimates tended to decline with an increase in nitrogen use for each crop. ${ }^{2}$ This suggests that RA constraints are important in determining skewness.

A sample of more recent studies includes Gallagher (1987) for U.S.-level soybean yields, 1941-1984, Nelson and Preckel (1989) and Nelson (1990) for Iowa corn in Iowa, 19611970, Swinton and King (1991) for Minnesota corn, 1944-1987, and Moss and Shonkwiler (1993) for U.S.-level corn, 1930-1990. All have found evidence of negative skewness. Joernsgaard and Halmoe (2003) considered intrafield yield distributions for linseed, rape, barley, and wheat in the United Kingdom and Denmark to find generally negative skewness where skewness declined with an increase in mean field yield.

However, Just and Weninger (1999) have raised methodology concerns with much of this large body of work. Data aggregation across space and possible misspecification of control factors (including time) may occur. In addition, they have expressed disquiet about how significance tests on normality have been presented and/or interpreted. This is relevant because it was also asserted in Just and Weninger that crop yield statistics, being averages over space and perhaps over time too, should comply with a relevant CLT whenever theorem assumptions apply.

Bear in mind that the limiting distribution for the mean of independent draws (independence requirement) from a common distribution (homogeneity requirement) is just the true distribution mean with zero values for all centered higher order moments. This is due to the strong law of large numbers under mild regularity conditions (Durrett 1996, p. 56). CLTs convey the way in which convergence to this distribution mean occurs, and scaling by root sample size $n^{0.5}$ is necessary to avoid a degenerate limiting distribution. Stated differently, if reported crop yield data are from a data process and at a level of aggregation such that one might reasonably expect some CLT to apply, then the observed distribution should have almost zero variance.

Endeavoring to control for criticisms of methodologies, Atwood, Shaik, and Watts (2002) tested and found evidence against normality for farm-level data on various crops and U.S. states, 1988-1997. Atwood, Shaik, and Watts (2003) rejected normality for farm-level corn, sorghum, and wheat in Kansas. Both

\footnotetext{
${ }^{2}$ See his table 3 on p. 722.
} 
studies identified significant negative skewness. Ramirez, Misra, and Field (2003) have discerned negative skewness for Iowa corn and soybeans using annual average data over 1950-1999, and positive skewness for Texas Plains dryland cotton, 1970-1999. Sherrick et al. (2004), with University of Illinois data 1992-1999, have found very suggestive evidence for negative skewness in corn and soybean yields.

In conclusion, although the methods may have been flawed in certain ways, the variety in crop data sets studied years of observation, and techniques used suggest the existence of nonzero skewness. For Corn Belt corn and soybeans and more recent data, evidence points strongly toward negative skewness. While accepting the relevance of CLTs and other statistical laws, we set aside aggregation issues by considering a small, homogeneous plot so that macrolevel aggregation under independent or near independent draws can be ignored.

This article will address the technical implications of the LoM technology in the presence of stochastic RAs. Three statistical models of RA distributions are considered, the uniform, the normal, and powers of the raised cosine. These distributions have null skew in order to avoid introducing bias. It is shown that both positive and negative yield skewness can be supported. Mathematical analysis and simulation methods are used to explore how heterogeneity in the means and variances of RAs act to modify yield skewness.

It is argued too that the empirical observations of typically negative crop yield skew for prime agricultural cropland may be partly due to left-tail effects of the LoM. In intensively cultivated areas where most inputs can be controlled with some precision then one might expect thin left tails on the RA distributions, and so negatively skewed yield. Irrigation, on the other hand, places a floor on a RA and could promote positive skewness. In addition to the above, weather-conditioned yield skewness is related to unconditional skewness to show that they differ by a term involving how yield mean and yield variance covary over weather realizations.

\section{Framework}

The LoM yield technology for RAs $\varepsilon_{i} \in\left[0, \varepsilon_{i}^{u}\right]$ $\subset \mathbb{R}_{+}, i \in \Omega_{N} \equiv\{1,2, \ldots, N\}$, specifies yield as

$$
y=\min \left[\varepsilon_{1}, \ldots, \varepsilon_{N}\right] .
$$

This expression is very general because the distribution of $\varepsilon=\left(\varepsilon_{1}, \ldots, \varepsilon_{N}\right) \subset \mathbb{R}_{+}^{N}$ is determined by the market input vector $x \in \overline{\mathbb{R}}_{+}^{M}$, among other factors, where $\overline{\mathbb{R}}_{+}^{M}$ is the positive closed $M$-dimensional orthant of reals. Market inputs are enumerated as $x_{j}, j \in\{1,2, \ldots$, $M\} \equiv \Omega_{M}$. The upper bound on each RA, $\varepsilon_{i}^{u}$, is assumed fixed for convenience as it will not be relevant to our analysis, and we define $y^{u}=\max _{i \in \Omega_{N}}\left\{\varepsilon_{i}^{u}\right\}$. The unit output price is $P$. Factor prices are $w_{j}$ where $w \in \overline{\mathbb{R}}_{+}^{M}$ represents the vector of factor prices.

If the $\varepsilon_{i}$ are random, then equation (1) provides the first-, or least-, order statistic (David and Nagaraja 2003). Model the $\varepsilon_{i}$ as being independent with input-conditioned distributions $F^{i}\left(\varepsilon_{i} \mid x\right)$ and densities $f^{i}\left(\varepsilon_{i} \mid x\right)$ where all are continuously differentiable in $x$. Survival functions are $\bar{F}^{i}\left(\varepsilon_{i} \mid x\right)=1-F^{i}\left(\varepsilon_{i} \mid x\right)$, and the general formula for the cumulative distribution of $y$ is

$$
\begin{aligned}
& G(y \mid x) \\
& \quad=\operatorname{Prob}\left[\varepsilon_{1} \leq y \text { or } \ldots \text { or } \varepsilon_{N} \leq y \mid x\right] \\
& \quad=1-\operatorname{Prob}\left[\varepsilon_{1}>y, \varepsilon_{2}>y, \ldots, \varepsilon_{N}>y \mid x\right] \\
& \quad=1-\bar{G}(y \mid x) ; \quad \bar{G}(y \mid x)=\prod_{j=1}^{N} \bar{F}^{j}(y \mid x) .
\end{aligned}
$$

Its probability density function is

$$
\begin{aligned}
g(y) & =\frac{d[1-\bar{G}(y \mid x)]}{d y} \\
& =-\frac{d \prod_{j=1}^{N} \bar{F}^{j}(y \mid x)}{d y} \\
& =\sum_{i=1}^{N}\left(\prod_{\substack{j=1, j \neq i}}^{N} \bar{F}^{j}(y \mid x)\right) f^{i}(y \mid x) \\
& =\sum_{i=1}^{N}\left(\prod_{j=1}^{N} \bar{F}^{j}(y \mid x)\right)\left(\frac{f^{i}(y \mid x)}{\bar{F}^{i}(y \mid x)}\right) \\
& =\bar{G}(y \mid x) \sum_{i=1}^{N} \frac{f^{i}(y \mid x)}{\bar{F}^{i}(y \mid x)} .
\end{aligned}
$$

Expected profit is assumed to be strictly concave in $x$, and may be written as 
(4)

$$
\begin{aligned}
& V(x ; P, w) \\
& \quad=P E[y \mid x]-\sum_{k=1}^{M} w_{k} x_{k} \\
& \quad=P \int_{0}^{y^{u}} \bar{G}(y \mid x) d y-\sum_{k=1}^{M} w_{k} x_{k}
\end{aligned}
$$

where we have computed the inputconditioned expected yield as

$$
\begin{aligned}
E[y \mid x] & =\int_{0}^{y^{u}} y d G(y \mid x) \\
& =\left.y G(y \mid x)\right|_{0} ^{y^{u}}-\int_{0}^{y^{u}} G(y \mid x) d y \\
& =\int_{0}^{y^{u}} \bar{G}(y \mid x) d y .
\end{aligned}
$$

Thus, the optimality conditions are

$$
\begin{aligned}
\frac{d V(x ; P, w)}{d x_{k}} & P \frac{d E[y \mid x]}{d x_{k}}-w_{k} \\
= & P \int_{0}^{y^{u}} \frac{d \bar{G}(y \mid x)}{d x_{k}} d y-w_{k} \\
= & P \int_{0}^{y^{u}} \frac{d \prod_{j=1}^{N} \bar{F}^{j}(y \mid x)}{d x_{k}} d y-w_{k} \\
= & P \int_{0}^{y^{u}} \prod_{j=1,}^{N} \bar{F}^{j}(y \mid x) \\
& \times \sum_{i=1}^{N}\left(\frac{\partial \bar{F}^{i}(y \mid x)}{\partial x_{k}}\right) d y-w_{k} \\
= & P \int_{0}^{y^{u}} \bar{G}(y \mid x) \sum_{i=1}^{N}\left(\frac{\partial \bar{F}^{i}(y \mid x) / \partial x_{k}}{\bar{F}^{i}(y \mid x)}\right) d y \\
& -w_{k}=0 \quad \forall k \in \Omega_{M}
\end{aligned}
$$

with solution arguments $x_{k}^{*}(P, w)$. Having characterized input choices we will henceforth take them as given and fixed, focusing instead on the yield distribution they determine. The uniform, normal, and power-raised cosine RA distributions will be considered in that sequence.

\section{Skewness and the Uniform Case}

The yield distribution of interest is when inputs are fixed, for otherwise the measured statistical attributes of yield may be due to heterogeneity in input uses over the area of interest and not to the technology itself. To make further progress in this section, we also assume that each $\varepsilon_{i}$ is independently drawn from the standard continuous uniform distribution, $\varepsilon_{i} \sim$ $U[0,1]$. Thus there is no bias in favor of any resource and in addition the RAs are symmetric around the mean so that they have zero skewness. A well-known result is that the first-order statistic then has density

$$
g(y ; 1, N)=\frac{(1-y)^{N-1}}{\int_{0}^{1}(1-z)^{N-1} d z}
$$

or the beta distribution with parameters $\alpha=1$ and $\beta=N$ (Gupta and Nadarajah 2004a, p. 89). It is not consistent with the majority of yield distributions estimated in the literature.

Note that $d g(y ; 1, N) / d y<0$, that is, the density is downward sloping wherever it has support so that positive skewness is to be expected. The mean, variance, and third central moment are $\mu_{y}=(1+N)^{-1}, \sigma_{y}^{2}=N(1+N)^{-2}(2+N)^{-1}$, and $\zeta_{y}=2 N(N-1)(2+N)^{-1}(3+N)^{-1} \times$ $(1+N)^{-3}>0$. Bearing in mind that skewness is defined as $\gamma_{y}=\zeta_{y} / \sigma_{y}^{3}$, we have

$$
\gamma_{y}=\frac{2(N-1)}{(3+N)} \sqrt{\frac{2+N}{N}}>0
$$

Thus, yield in this case expresses positive skew as found in Day (1965) for Mississippi cotton and corn, 1921-1957 and Ramirez, Misra, and Field (2003) for Texas Dryland cotton, 19701999.

\section{Heterogeneity in Means}

Of course, in reality even if RAs have uniform marginals, they are unlikely to have common means or variances, if only because of heterogeneous factor prices and technological capabilities across RAs. Neither are the RAs likely to be independent. We will relax each of these conditions in turn. To focus on effects, let there be just two resources at issue where $\varepsilon_{1}$ is discrete uniformly distributed on point pair $\{0,1\}, \varepsilon_{2}$ is discrete uniformly distributed on $\{\tau, 1+\tau\}, \tau \in[0,1]$, and these random 
variables are independent. ${ }^{3}$ Therefore, the random variables have the same higher central moments for marginals, differing only by the shifted mean. In order to commence with a zero skew distribution, let the probability of both low and high states be 0.5 .

The distribution of $y=\min \left[\varepsilon_{1}, \varepsilon_{2}\right]$ is 0 with probability $0.5, \tau$ with probability 0.25 , and 1 with probability 0.25 . The moments are $\mu_{y}=(\tau+1) / 4, \sigma_{y}^{2}=\left(3 \tau^{2}-2 \tau+3\right) / 16, \zeta_{y}=$ $3(1-\tau)^{2}(1+\tau) / 32$ with skewness $\gamma_{y}=$ $6(1-\tau)^{2}(1+\tau)\left(3 \tau^{2}-2 \tau+3\right)^{-3 / 2}>0$ where the derivative of interest satisfies

$$
\frac{d \gamma_{y}}{d \tau}=-\frac{96(1-\tau) \tau}{\left(3 \tau^{2}-2 \tau+3\right)^{2.5}}<0
$$

on $\tau \in[0,1]$. Omitted computations here and elsewhere in this article are available in Hennessy (2008). Thus, heterogeneity in location alone tends to reduce (positive) skewness for the discrete uniform distribution. This is because the location shift takes probability mass away from a support point at the lower end of the distribution.

\section{Heterogeneity in Variances}

As above, let there be just two resources at issue where $\varepsilon_{1}$ is discrete uniformly distributed on $\{0,1\}$. But let $\varepsilon_{2}$ be discrete uniformly distributed on $\{\tau, 1-\tau\}, \tau \in[0,0.5]$, while these random variables are (again) independent. If $\tau=0.5$ then the support of $\varepsilon_{2}$ is concentrated at $\varepsilon_{2}=0.5$. As before, set the skew as zero by letting the probability of the low state be 0.5 in each case. The distribution of $y=\min \left[\varepsilon_{1}, \varepsilon_{2}\right]$ is 0 with probability $0.5, \tau$ with probability 0.25 , and $1-\tau$ with probability 0.25 . The moments are $\mu_{y}=0.25, \sigma_{y}^{2}=\left(8 \tau^{2}-8 \tau+3\right) / 16$, and $\zeta_{y}=3(1-2 \tau)^{2} / 32$ with skewness $\gamma_{y}=6(1-$ $2 \tau)^{2}\left(8 \tau^{2}-8 \tau+3\right)^{-3 / 2}$ so that the derivative of interest is

(10)

$$
\frac{d \gamma_{y}}{d \tau}=\frac{96(2 \tau-1) \tau(1-\tau)}{\left(3-8 \tau+8 \tau^{2}\right)^{2.5}} \stackrel{\text { sign }}{=} \tau-0.5 \leq 0 .
$$

Heterogeneity in variance, through contracting the support of one distribution, reduces skewness. As with a location shift, heterogeneity takes probability mass away from a heavily weighted support point on the distribution's left tail.

\footnotetext{
${ }^{3}$ If $\tau>1$ then $\min \left[\varepsilon_{1}, \varepsilon_{2}\right] \equiv \varepsilon_{1}$.
}

\section{Dependence}

Following Dasgupta and Maskin (1987), set

$$
\begin{aligned}
& \left(\varepsilon_{1}, \varepsilon_{2}\right) \\
& \quad=\left\{\begin{array}{lll}
(0,0) & \text { with probability } & 0.25(1+\rho) ; \\
(0,1) & \text { with probability } & 0.25(1-\rho) ; \\
(1,0) & \text { with probability } & 0.25(1-\rho) ; \\
(1,1) & \text { with probability } & 0.25(1+\rho) ;
\end{array}\right.
\end{aligned}
$$

for $\rho \in[-1,1]$. When $\rho>0$ then there is more probability on points $(0,0)$ and $(1,1)$, and this creates positive dependence. In fact, $\operatorname{Cov}\left(\varepsilon_{1}, \varepsilon_{2}\right)=E\left[\varepsilon_{1} \varepsilon_{2}\right]-E\left[\varepsilon_{1}\right] E\left[\varepsilon_{2}\right]=$ $0.25(1+\rho)-(0.5)(0.5)=0.25 \rho$. Mean, variance, and third central moment of the least-order statistic (i.e., yield) are now $\mu_{y}=0.25(1+\rho), \sigma_{y}^{2}=(3-\rho)(1+$ $\rho) / 16$, and $\zeta_{y}=(3-\rho)\left(1-\rho^{2}\right) / 32$ where skewness is $\gamma_{y}=2(1-\rho)(3-\rho)^{-0.5}(1+$ $\rho)^{-0.5} \geq 0$. Note that $\operatorname{Lim}_{\rho \downarrow-1} \gamma_{y}=+\infty$ and $\gamma_{y}=0$ when $\rho=1$.

The derivative with respect to the correlation parameter is

$$
\frac{d \gamma_{y}}{d \rho}=-\frac{8}{(3-\rho)^{3 / 2}(1+\rho)^{3 / 2}}<0 .
$$

So an increase in correlation decreases skewness for the discrete uniform distribution. As correlation increases then the distribution of $\min \left[\varepsilon_{1}, \varepsilon_{2}\right]$ becomes more like the uniform distribution, with zero skewness. A decrease in correlation can be seen as an increase in heterogeneity among RAs. Overall, we see that skewness falls when means diverge (more heterogeneity), one of the random variables assumes less variance (more heterogeneity), or the distributions become more correlated (less heterogeneity).

\section{Skewness and the Normal Case}

Now let the $\varepsilon_{i}$ be independently drawn from an identical distribution, namely $F^{i}\left(\varepsilon_{i}\right)=\Phi\left(\left(\varepsilon_{i}-\right.\right.$ $\mu) / \sigma)$, the standard cumulative normal before being relocated and scaled by common parameters. Then the yield density for the minimum of $N$ draws is

$$
g(y)=\frac{N}{\sigma}\left[1-\Phi\left(\frac{y-\mu}{\sigma}\right)\right]^{N-1} \phi\left(\frac{y-\mu}{\sigma}\right)
$$


where $\phi(\cdot)$ is the density of the normalized distribution and $1-\Phi(\cdot)$ is the survival function for some RA. This is an instance of the betanormal distribution as discussed in Eugene, Lee, and Famoye (2002). ${ }^{4}$ When $N=2$ then Choi (2005), correcting Gupta and Nadarajah (2004b), shows the first three moments to be $\mu_{y}=\mu-\sigma \pi^{-0.5}<\mu, \sigma_{y}^{2}=(\pi-1) \sigma^{2} \pi^{-1}$, and $\zeta_{y}=0.5(\pi-4) \sigma^{3} \pi^{-3 / 2}<0$. Thus, and by contrast with the uniform distribution, skewness is negative at

$$
\gamma_{y}=\frac{\pi-4}{2(\pi-1)^{3 / 2}}
$$

This should not be surprising when one considers how weighting $1-\Phi((y-\mu) / \sigma)$ biases density $\phi((y-\mu) / \sigma)$ in equation (13). Though low at low yields, the yield density function should not be as low for low-yield draws as for high-yield draws. This is by contrast with uniform RA densities, where the survival function density in equation (7) completely determines the shape of yield density, and (implausibly) yield density has negative derivative everywhere it has support. A comparison of equation (7) with equation (13) suggests an important role for the left tails of RAs in determining yield skewness under the LoM technology.

To illustrate the effects of RA heterogeneity on the yield distribution, let $N=2$ and suppose first that the random variables are perfectly positively correlated. Then yield follows the standard normal up to location and scale, thus having zero skew. Suppose instead that the random variables have perfect negative correlation so that $\left(\varepsilon_{1}-\mu\right) / \sigma \equiv-\left(\varepsilon_{2}-\mu\right) / \sigma$. Then the yield distribution is half-normal with support to the left of $\mu$, and clearly has negative skew. By contrast with the uniform, an increase in correlation (and so a decrease in heterogeneity) from $\rho=0$ to $\rho=1$ increases skewness to $\gamma_{y}=0$.

The case of drawing from a bivariate normal with heterogeneous means involves a nonmonotonicity. If, under independence and equal variances, means differ substantially then only the marginal with the lower mean matters and skewness should not differ much from zero. With means $\bar{\varepsilon}_{1}$ and $\bar{\varepsilon}_{2}$, as $\bar{\varepsilon}_{1}$ increases

\footnotetext{
${ }^{4}$ Actually, as has been recently pointed out by Jones (2004), when $N$ is a natural number then this is the least-order statistic of independent draws from a common normal distribution. The literature on order statistics for i.i.d. normal random variables has a long pedigree dating at least as far back as Bose and Gupta (1959).
}

toward $\bar{\varepsilon}_{2}$ then yield skewness should decrease away from zero until $\bar{\varepsilon}_{1}=\bar{\varepsilon}_{2}$ and should increase toward zero thereafter. So heterogeneity in means should increase skewness toward zero. This is by contrast with the uniform case where heterogeneity in means decreases skewness toward zero.

Alternatively, suppose we allow possibly distinct means and variances on the RAs where the variance of $\varepsilon_{2}$ recedes to zero. Perhaps this results from tighter control of nitrogen available for crop growth as a result of using soil nitrogen tests before application. Of course, for $\varepsilon_{2}$ constant then correlation between $\varepsilon_{1}$ and $\varepsilon_{2}$ is not a meaningful statistic, and it may be ignored. With mean of $\varepsilon_{2}$ at $\bar{\varepsilon}_{2}$, the yield distribution becomes censored normal with support to the left of $\bar{\varepsilon}_{2}$ and strictly positive probability massed at $\bar{\varepsilon}_{2}$. Again, the distribution is negatively skewed. So casual heuristic reasoning suggests that heterogeneity through lower variance for one RA should introduce downward bias in the skewness of the least-order statistic of symmetric random variables when compared with the baseline case where the RA distributions are independent and identical. This downward bias is as under the comparable scenario for the uniform distribution.

Unfortunately, thought exercises such as the above aside, little appears to be known about order statistics for draws from non-i.i.d. normal distributions. To investigate further, table 1 provides moment estimates for a variety of scenarios when $N=2$. The benchmark is italicized and bolded with means at $\bar{\varepsilon}_{1}=\bar{\varepsilon}_{2}=10$, standard deviations at $\sigma_{1}=\sigma_{2}=1$ where $\sigma_{i}^{2}=$ $\operatorname{Var}\left(\varepsilon_{i}\right)$, and correlation at $\rho=0$. After taking 10,000 independent draws, antithetic variates were used to double the sample to $20,000 .^{5}$

Note first that the mean and standard deviation of yield increase with an increase in correlation. For yield mean, per equation (1), it is preferable that low draws tend to come in pairs in order to get them out of the way. For standard deviation, the LoM minimization operation pushes probability weightings toward the lower end of the support so that one should expect dispersion to decline relative to the underlying RA distributions. Notice also that skewness is never positive, i.e., the simulations suggest that what applies for the

\footnotetext{
${ }^{5}$ For antithetic variates, if the vector $\left(\varepsilon_{1}, \varepsilon_{2}\right)=\left(\bar{\varepsilon}_{1}, \bar{\varepsilon}_{2}\right)+\left(\widehat{\varepsilon}_{1}-\right.$ $\left.\bar{\varepsilon}_{1}, \widehat{\varepsilon}_{2}-\bar{\varepsilon}_{2}\right)$ is drawn then so is $\left(\varepsilon_{1}, \varepsilon_{2}\right)=\left(\bar{\varepsilon}_{1}, \bar{\varepsilon}_{2}\right)-\left(\widehat{\varepsilon}_{1}-\bar{\varepsilon}_{1}, \widehat{\varepsilon}_{2}-\right.$ $\left.\bar{\varepsilon}_{2}\right)$. The intent is to promote law-of-large-numbers convergence by balanced sampling (Boyle, Broadie, and Glasserman 1997).
} 
Table 1. Yield Moments When Resource Availabilities are Jointly Normally Distributed

\begin{tabular}{|c|c|c|c|c|c|c|}
\hline $\begin{array}{l}\text { RA } \\
\text { Means }\end{array}$ & $\begin{array}{c}\text { Yield } \\
\text { Moments }\end{array}$ & $\begin{array}{l}\left(\sigma_{1}, \sigma_{2}\right) \\
=(1,1)\end{array}$ & $\begin{array}{l}\left(\sigma_{1}, \sigma_{2}\right) \\
=(1,2)\end{array}$ & $\begin{array}{l}\left(\sigma_{1}, \sigma_{2}\right) \\
=(2,2)\end{array}$ & $\begin{array}{c}\left(\sigma_{1}, \sigma_{2}\right) \\
=(1,0.5)\end{array}$ & $\begin{aligned} & \left(\sigma_{1}, \sigma_{2}\right) \\
= & (0.5,0.5)\end{aligned}$ \\
\hline \multirow{3}{*}{$\left(\bar{\varepsilon}_{1}, \bar{\varepsilon}_{2}\right)=(10,10)$} & Mean & 9.435 & 9.108 & $\begin{array}{l}\rho=0 \\
8.864\end{array}$ & 9.550 & 9.719 \\
\hline & Std. Dev. & 0.827 & 1.303 & 1.642 & 0.654 & 0.416 \\
\hline & Skewness & -0.131 & -0.664 & -0.177 & -0.671 & -0.144 \\
\hline \multirow{3}{*}{$\left(\bar{\varepsilon}_{1}, \bar{\varepsilon}_{2}\right)=(8,10)$} & Mean & 7.950 & 7.780 & 7.593 & 7.983 & 7.999 \\
\hline & Std. Dev. & 0.959 & 1.020 & 1.751 & 0.963 & 0.500 \\
\hline & Skewness & -0.122 & -0.246 & -0.191 & -0.149 & -0.010 \\
\hline \multirow{3}{*}{$\left(\bar{\varepsilon}_{1}, \bar{\varepsilon}_{2}\right)=(12,10)$} & Mean & 9.949 & 9.782 & 9.609 & 9.985 & 10.000 \\
\hline & Std. Dev. & 0.938 & 1.696 & 1.744 & 0.492 & 0.500 \\
\hline & Skewness & -0.144 & -0.448 & $\begin{array}{c}-0.176 \\
\rho=0.5\end{array}$ & -0.029 & -0.011 \\
\hline \multirow[t]{3}{*}{$\left(\bar{\varepsilon}_{1}, \bar{\varepsilon}_{2}\right)=(10,10)$} & Mean & 9.600 & 9.300 & 9.201 & 9.653 & 9.800 \\
\hline & Std. Dev. & 0.912 & 1.420 & 1.829 & 0.707 & 0.459 \\
\hline & Skewness & -0.028 & -0.561 & -0.042 & -0.623 & -0.041 \\
\hline \multirow[t]{3}{*}{$\left(\bar{\varepsilon}_{1}, \bar{\varepsilon}_{2}\right)=(8,10)$} & Mean & 7.990 & 7.894 & 7.826 & 7.997 & 8.000 \\
\hline & Std. Dev. & 1.000 & 1.077 & 1.908 & 0.999 & 0.499 \\
\hline & Skewness & -0.022 & -0.229 & -0.049 & -0.052 & 0.000 \\
\hline \multirow{4}{*}{$\left(\bar{\varepsilon}_{1}, \bar{\varepsilon}_{2}\right)=(12,10)$} & Mean & 9.991 & 9.895 & 9.830 & 9.997 & 10.000 \\
\hline & Std. Dev. & 0.988 & 1.835 & 1.915 & 0.503 & 0.504 \\
\hline & Skewness & -0.022 & -0.272 & -0.056 & -0.006 & 0.000 \\
\hline & & & & $\rho=-0.5$ & & \\
\hline \multirow{3}{*}{$\left(\bar{\varepsilon}_{1}, \bar{\varepsilon}_{2}\right)=(10,10)$} & Mean & 9.305 & 8.933 & 8.605 & 9.470 & 9.685 \\
\hline & Std. Dev. & 0.721 & 1.179 & 1.452 & 0.584 & 0.361 \\
\hline & Skewness & -0.341 & -0.923 & -0.365 & -0.935 & -0.375 \\
\hline \multirow{3}{*}{$\left(\bar{\varepsilon}_{1}, \bar{\varepsilon}_{2}\right)=(8,10)$} & Mean & 7.894 & 7.661 & 7.379 & 7.963 & 7.997 \\
\hline & Std. Dev. & 0.885 & 0.934 & 1.555 & 0.936 & 0.488 \\
\hline & Skewness & -0.335 & -0.463 & -0.379 & -0.275 & -0.064 \\
\hline \multirow{3}{*}{$\left(\bar{\varepsilon}_{1}, \bar{\varepsilon}_{2}\right)=(12,10)$} & Mean & 9.891 & 9.652 & 9.398 & 9.963 & 9.996 \\
\hline & Std. Dev. & 0.880 & 1.592 & 1.537 & 0.473 & 0.494 \\
\hline & Skewness & -0.341 & -0.658 & -0.416 & -0.148 & -0.070 \\
\hline
\end{tabular}

first-order statistic with i.i.d. normal draws may be robust to relaxing the i.i.d. requirements.

Confining attention now to zero correlation simulations, yield skewness generally does not differ much across differences in means when variances are common. Such differences in mean may be due to soil and weather endowment heterogeneities or price differences in nutrient markets. Skewness tends to be more strongly negative when the common variance is large. Heterogeneity in means only ensures that the distribution with the lower mean dominates when determining the first-order statistic. With a sufficiently large gap in means and sufficiently low standard deviations, the yield skew will be close to zero. When $\left(\bar{\varepsilon}_{1}, \bar{\varepsilon}_{2}\right)=$ $(8,10)$ and $\left(\sigma_{1}, \sigma_{2}\right)=(0.5,0.5)$ then skewness is -0.01 .

For $\bar{\varepsilon}_{1}=\bar{\varepsilon}_{2}=10$, and recalling that skewness is normalized by yield variance, yield skewness is more negative when there is also heterogeneity in the RA variances. This is as with the uniform distribution, but the point bears elaboration. A contraction in the variance of one RA ensures that the upper tail of the yield distribution is increasingly concentrated around the mean of that RA. An increase in the RA variance would not have that effect. For normally distributed RAs with equal means, a decrease in the variance of one tends to induce a more negative skew because one is converging on a censored (from the top) normal yield distribution. An increase in the variance of one RA also tends to induce a more negative skew. Then the more dispersed distribution dominates in determining the left end of yield density whereas the tightly dispersed distribution dominates in determining the right end of yield density.

The case $\left(\rho, \sigma_{1}, \sigma_{2}\right)=(0,1,2)$ merits attention. There, skewness is -0.664 under $\bar{\varepsilon}_{1}=$ $\bar{\varepsilon}_{2}=10,-0.246$ under $\left(\bar{\varepsilon}_{1}, \bar{\varepsilon}_{2}\right)=(8,10)$ and -0.448 under $\left(\bar{\varepsilon}_{1}, \bar{\varepsilon}_{2}\right)=(12,10)$. Skewness becomes less negative when $\bar{\varepsilon}_{1}$ decreases from 10 
to 8 and when $\bar{\varepsilon}_{1}$ increases from 10 to 12 . At $\bar{\varepsilon}_{1}=8$ then $\varepsilon_{1}$ is dominant in determining yield because it has lower mean and lower variance. So skewness should move toward 0 , that of the univariate normal distribution. The move toward 0 skewness is not as pronounced when $\bar{\varepsilon}_{1}$ changes from 10 to 12 because the large variance of $\varepsilon_{2}$ ensures it can still throw up a good draw such that $\varepsilon_{1}$ narrowly distributed at the upper end of the yield range will still be important.

Moving to positive correlation, with $\bar{\varepsilon}_{1}=\bar{\varepsilon}_{2}=10$ then the effect on skewness is clear. When compared with $\rho=0$, skewness is always less negative. On the other hand, negative correlation makes skewness even more negative for any given set of RA mean and standard deviation parameters. The most negative skew statistic arises when $\left(\rho, \bar{\varepsilon}_{1}, \bar{\varepsilon}_{2}, \sigma_{1}, \sigma_{2}\right)=(-0.5,10,10,1,0.5)$, followed closely by $\left(\rho, \bar{\varepsilon}_{1}, \bar{\varepsilon}_{2}, \sigma_{1}, \sigma_{2}\right)=$ $(-0.5,10,10,1,2)$. Here, the common mean ensures that both marginals are relevant. The negative correlation ensures that a moderately below average draw from one marginal is very likely to matter, thus facilitating a pileup of probability mass toward the RA means. Variance heterogeneity allows for a thinly spread out left tail to the yield distribution.

\section{Skewness and the Raised Power Cosine Case}

To further probe the conjecture that distribution tails determine skewness, consider the raised cosine distribution. Let $y=\min \left[\varepsilon_{1}, \varepsilon_{2}\right]$ where the $\varepsilon_{i}$ are independent with common density

$$
f\left(\varepsilon_{i}\right)=0.5 \operatorname{Cos}\left(\varepsilon_{i}\right), \quad \varepsilon_{i} \in[-\pi / 2, \pi / 2]
$$

that is, on the cosine function's domain of positive value. ${ }^{6}$ The distribution is chosen because the power transformation $0.5 \operatorname{Cos}\left(\varepsilon_{i}\right) \rightarrow$ $\kappa(\alpha)\left[\operatorname{Cos}\left(\varepsilon_{i}\right)\right]^{\alpha}$ thins out the density tails when $\alpha>1$, because densities in this class have zero skewness for all $\alpha>0$, and also because the class of densities is analytically tractable. The cumulative distribution for RA density $f\left(\varepsilon_{i}\right)=$ $0.5 \operatorname{Cos}\left(\varepsilon_{i}\right)$ is $F\left(\varepsilon_{i}\right)=\left[1+\operatorname{Sin}\left(\varepsilon_{i}\right)\right] / 2$. From equation (3),

\footnotetext{
${ }^{6}$ Here, 0.5 normalizes since $\int_{-\pi / 2}^{\pi / 2} \operatorname{Cos}\left(\varepsilon_{i}\right) d \varepsilon_{i}=\left.\operatorname{Sin}\left(\varepsilon_{i}\right)\right|_{-\pi / 2} ^{\pi / 2}=$ 2 . The support was chosen for analytic convenience. While it intersects the negative domain, remember that skewness is locationindependent. A location shift of support from $[-\pi / 2, \pi / 2]$ to $[\tau-\pi / 2, \tau+\pi / 2], \tau \geq-\pi / 2$, would not affect skewness.
}

$$
g(y)=\frac{\operatorname{Cos}(y)[1-\operatorname{Sin}(y)]}{2}
$$

with moments $\mu_{y}=-0.393, E\left[y^{2}\right]=0.467$, $E\left[y^{3}\right]=-0.380, \gamma_{y}=E\left[\left(y-\mu_{y}\right)^{3}\right] / \sigma_{y}^{3}=0.283 .^{7}$

Instead, suppose the $\varepsilon_{i}$ are independent with common density $(0.5 \pi)^{-1} \operatorname{Cos}^{2}\left(\varepsilon_{i}\right)$ on $[-\pi / 2$, $\pi / 2$ ], the cosine function's domain of positive value. ${ }^{8}$ Squaring the smaller values close to the support boundaries thins out the tails. The cumulative distribution for an RA is $0.5+\left[\varepsilon_{i}+\right.$ $\left.\operatorname{Cos}\left(\varepsilon_{i}\right) \operatorname{Sin}\left(\varepsilon_{i}\right)\right] / \pi$. From equation (3), yield density is

$g(y)$

$$
=\frac{4 \operatorname{Cos}^{2}(y)\{0.5-([y+\operatorname{Cos}(y) \operatorname{Sin}(y)]) / \pi\}}{\pi}
$$

while moments are $\mu_{y}=-0.325, E\left[y^{2}\right]=0.323$, $E\left[y^{3}\right]=-0.230, \gamma_{y}=0.156$. Skewness, though still positive, has declined.

To show that the decline is due to a thinned out tail on the yield distribution, consider table 2 in which moments for densities are given when $\alpha \in\{1,2, \ldots, 9\} \cup\{50\} .{ }^{9}$ Observe that mean yield increases and yield variance declines with an increase in the value of $\alpha$. When the value of $\alpha$ increases then each RA distribution is more peaked around its mean, RA variance declines, and so low-yield draws become less likely. Yield skewness declines with an increase in $\alpha$ until it becomes 0 at approximately $\alpha=6$ and somewhat negative thereafter. The $\alpha=50$ simulation shows that skewness never becomes markedly negative.

\section{Conditional Skewness and Overall Skewness}

We turn next to how the measurement of skewness can be affected by the presence of different production environments. This setting was presented in Ker and Goodwin (2000) to explain left-tail behavior when yield distributions are normal conditional on weather,

\footnotetext{
${ }^{7}$ Integrations were performed with the assistance of the Wolfram Integrator webMATHEMATICA, available at http://integrals.wolfram.com/index.jsp.

${ }^{8}$ The normalizing constant is $0.5 \pi$ since $\int_{-\pi / 2}^{\pi / 2} \operatorname{Cos}^{2}\left(\varepsilon_{i}\right) d \varepsilon_{i}=$ $\left.0.5\left[\varepsilon_{i}+\operatorname{Cos}\left(\varepsilon_{i}\right) \operatorname{Sin}\left(\varepsilon_{i}\right)\right]\right|_{-\pi / 2} ^{\pi / 2}=0.5 \pi$.

${ }^{9}$ Analytic integrations were conducted for $\alpha \in\{1,2,3,4\}$, and are available in Hennessy (2008). Results for $\alpha \in\{5,6,7,8,9\} \cup$ $\{50\}$ were arrived at using Monte Carlo simulations with 10,000 draws, which were doubled to 20,000 upon use of antithetic variates. Skewness, though not mean or variance, proved to be somewhat unstable so the numbers reported are averages for 10 runs. A reviewer's comments on simulations over raised power cosine distributions are greatly appreciated.
} 
Table 2. Yield Moments When Resource Availabilities Are Power-Raised Cosine-Distributed

Yield

\begin{tabular}{lrrrrrrrrrr} 
Moments & $\alpha=1$ & $\alpha=2$ & $\alpha=3$ & $\alpha=4$ & $\alpha=5$ & $\alpha=6$ & $\alpha=7$ & $\alpha=8$ & $\alpha=9$ & $\alpha=50$ \\
\hline Mean & -0.393 & -0.325 & -0.282 & -0.247 & -0.231 & -0.214 & -0.200 & -0.188 & -0.180 & -0.079 \\
Std. Dev. & 0.559 & 0.466 & 0.407 & 0.369 & 0.335 & 0.310 & 0.287 & 0.275 & 0.259 & 0.116 \\
Skewness & 0.283 & 0.156 & 0.084 & 0.015 & 0.009 & -0.002 & -0.004 & -0.005 & -0.008 & -0.005
\end{tabular}

but where weather is not normally distributed. With yield as $y$ on support $y \in[0, \bar{y}]$, suppose the extent of rainfall (it could be soil nitrogen) is given as $\theta$ on support $\theta \in[0, \bar{\theta}]$ where the joint density is $h(y, \theta)$. Here the origin of this distribution is being ignored, and so any skewness introduced by the LoM is taken as given. Let unconditional mean yield be $\mu$ while the $\theta$-conditioned mean is $\mu(\theta)$ with $H(y \mid \theta)$ as the conditional distribution and $J(\theta)$ as the unconditional distribution of rainfall. Given $\delta(\theta)=$ $\mu(\theta)-\mu$, then the unconditional, centered, third moment of yield is

$$
\begin{aligned}
\chi_{y} \equiv & \int_{0}^{\bar{\theta}} \int_{0}^{\bar{y}}(y-\mu)^{3} d H(y \mid \theta) d J(\theta) \\
= & \int_{0}^{\bar{\theta}} \int_{0}^{\bar{y}}[y-\mu(\theta)+\delta(\theta)]^{3} d H(y \mid \theta) d J(\theta) \\
= & \int_{0}^{\bar{\theta}}\left[\int_{0}^{\bar{y}}[y-\mu(\theta)]^{3} d H(y \mid \theta)\right] d J(\theta) \\
& +3 \int_{0}^{\bar{\theta}} \delta(\theta)\left[\int_{0}^{\bar{y}}[y-\mu(\theta)]^{2} d H(y \mid \theta)\right] d J(\theta) \\
& +3 \int_{0}^{\bar{\theta}}[\delta(\theta)]^{2} \overbrace{\int_{0}^{\bar{y}}[y-\mu(\theta)] d H(y \mid \theta)}^{=0} d J(\theta) \\
& +\int_{0}^{\bar{\theta}}[\delta(\theta)]^{3} \overbrace{0}^{\bar{y}} d H(y \mid \theta) d J(\theta) \\
= & \int_{0}^{\bar{\theta}}\left[\int_{0}^{\bar{y}}[y-\mu(\theta)]^{3} d H(y \mid \theta)\right] d J(\theta) \\
& +3 \int_{0}^{\bar{\theta}} \delta(\theta)\left[\int_{0}^{\bar{y}}[y-\mu(\theta)]^{2} d H(y \mid \theta)\right] d J(\theta) \\
& +\int_{0}^{\bar{\theta}}[\delta(\theta)]^{3} d J(\theta) .
\end{aligned}
$$

When all conditional distributions have zero skewness, or $\int_{0}^{\bar{y}}[y-\mu(\theta)]^{3} d H(y \mid \theta)=0 \forall \theta \in$ $[0, \bar{\theta}]$, then equation (18) becomes

$$
\begin{aligned}
\chi_{y}= & 3 \int_{0}^{\bar{\theta}} \delta(\theta) \operatorname{Var}(y \mid \theta) d J(\theta) \\
& +\int_{0}^{\bar{\theta}}[\delta(\theta)]^{3} d J(\theta) \\
= & 3 \int_{0}^{\bar{\theta}}[\mu(\theta)-\mu] \operatorname{Var}(y \mid \theta) d J(\theta) \\
& +\int_{0}^{\bar{\theta}}[\mu(\theta)-\mu]^{3} d J(\theta) .
\end{aligned}
$$

Apply the covariance relation $E\left[\varepsilon_{1} \varepsilon_{2}\right] \equiv$ $E\left[\varepsilon_{1}\right] E\left[\varepsilon_{2}\right]+\operatorname{Cov}\left(\varepsilon_{1}, \varepsilon_{2}\right)$ to write equation (19) as

$$
\begin{aligned}
\chi_{y}= & 3 \operatorname{Cov}[\mu(\theta)-\mu, \operatorname{Var}(y \mid \theta)] \\
& +\int_{0}^{\bar{\theta}}[\mu(\theta)-\mu]^{3} d J(\theta) .
\end{aligned}
$$

So equation (20) shows two ways in which a distribution with zero skewness for any given rainfall level might have nonzero overall skewness. Considering the case of negative overall skewness, view the last term in equation (20). One might have that expected yield $\mu(\theta)$ at some given rainfall level is itself a negatively skewed random variable over the rainfall distribution so that $0>\int_{0}^{\bar{\theta}}[\mu(\theta)-\mu]^{3} d J(\theta)$. The other way is that the rainfall-conditioned mean yield and the rainfall-conditioned variance of yield covary negatively as rainfall changes. For example, as seems quite plausible, an increase in rainfall could increase expected yield and decrease the variance of yield. Then the covariance in equation (20) is negative.

In summary, if $(i)$ yield has nonpositive skew for any given rainfall level, (ii) rainfall-conditioned expected yield has nonpositive skew over the rainfall distribution, and (iii) more rainfall increases mean yield while also decreasing yield variance, then the unconditional yield is negatively skewed. Separately, note that if yield is statistically independent of rainfall then both terms in equation (20) are zero and the unconditional yield distribution exhibits the same 
skewness as the rainfall-conditioned yield distributions.

\section{Dramatic Innovation}

Relation (20) is suggestive when viewed with an eye to technical change. Suppose that a lower limit on $\theta$ is introduced, say by introducing irrigation, then expression $\int_{0}^{\bar{\theta}}[\mu(\theta)-\mu]^{3} d J(\theta)$ should become less negative or more positive as the left tail of the water availability distribution will be truncated. The effect on $\operatorname{Cov}[\mu(\theta)-\mu, \operatorname{Var}(y \mid \theta)]$ is less clear. But if $(i) \mu(\theta)-\mu$ increases most strongly with an increase in $\theta$ when $\theta$ is low, and if $(i i)$ $\operatorname{Var}(y \mid \theta)$ decreases with an increase in $\theta$, then the covariance is likely to become less negative upon truncation. This suggests that some technology innovations can induce a more positive yield skew.

In order to be more definitive, suppose that water is the only constraint. Let yield equal water availability, which has distribution $\varepsilon \sim F(\varepsilon)$. The availability of water rights at maximum rate flow $\phi$ per year then places a floor on crop yield. Circumventing the explicit modeling of irrigation choices, we posit that yield is now $\max [\varepsilon, \phi]$. Of course, the innovation increases mean yield and decreases yield variance. Now, and by stark contrast with the effects of the LoM, the yield distribution has an accumulation at the lower tail and is likely to have positive skewness. So the effects of a technical innovation on skewness depend very much on context.

Example. Consider the standard uniform distribution on $[0,1]$ when truncated from below at $y=z$. The noncentral moments are $E[y \mid z]=\int_{0}^{z} z d y+\int_{z}^{1} y d y=\left(1+z^{2}\right) / 2, E\left[y^{2} \mid z\right]$ $=\int_{0}^{z} z^{2} d y+\int_{z}^{1} y^{2} d y=\left(1+2 z^{3}\right) / 3$, and $E\left[y^{3} \mid\right.$ $z]=\int_{0}^{z} z^{3} d y+\int_{z}^{1} y^{3} d y=\left(1+3 z^{4}\right) / 4$ so that

$\gamma_{y}$

$$
\begin{aligned}
& =\frac{E\left[y^{3} \mid z\right]-3 E[y \mid z] E\left[y^{2} \mid z\right]+2(E[y \mid z])^{3}}{\left(E\left[y^{2} \mid z\right]-(E[y \mid z])^{2}\right)^{1.5}} \\
& =\frac{\left(1-4 z+6 z^{2}-4 z^{3}+z^{4}\right) z^{2} 6 \sqrt{3}}{\left(1-6 z^{2}+8 z^{3}-3 z^{4}\right)^{1.5}} .
\end{aligned}
$$

Evaluation over domain $[0,1)$ shows the expression is never negative. So, as intuition might suggest, the floor on the RA distribution increases skewness to be positive.
On the other hand, suppose the technology does not truncate entirely on the left. In particular, suppose the innovation piles fraction $\lambda$ of the initial density below $z$ up at $z$, while fraction $1-\lambda$ remains where it was. Then the moments are $E[y \mid z]=$ $(1-\lambda) \int_{0}^{z} y d y+\lambda z^{2}+\int_{z}^{1} y d y=0.5\left(1+\lambda z^{2}\right)$, $E\left[y^{2} \mid z\right]=(1-\lambda) \int_{0}^{z} y^{2} d y+\lambda z^{3}+\int_{z}^{1} y^{2} d y=$ $\left(1+2 \lambda z^{3}\right) / 3$, and $E\left[y^{3} \mid z\right]=(1-\lambda) \int_{0}^{z} y^{3} d y+$ $\lambda z^{4}+\int_{z}^{1} y^{3} d y=\left(1+3 \lambda z^{4}\right) / 4$ so that

$$
=\frac{\left(1-4 z+3 \lambda z^{2}+3 z^{2}-4 \lambda z^{3}+\lambda^{2} z^{4}\right) \lambda z^{2} 6 \sqrt{3}}{\left(1-6 \lambda z^{2}+8 \lambda z^{3}-3 \lambda^{2} z^{4}\right)^{1.5}} .
$$

It has value 0 when $z=0$, positive value in the neighborhood of $z=0$, value $-\lambda^{2}(1+$ $3 \lambda)^{-1.5}(1-\lambda)^{-0.5} 6 \sqrt{3}<0$ when $z=1$, and value -0.5 when $(\lambda, z)=(1 / 3,1)$. In this case, the innovation's impact on skewness depends on how significant the innovation is. If it is modestly significant, with a low $z$ value, then the innovation could turn the null skew uniform yield distribution into one that is positively skewed. If the innovation has dramatic consequences, then skewness can become very negative.

\section{Conclusion}

Crop insurance markets and policies as well as other agricultural policies in many countries have generated needs for accurate models of crop yield distributions. Given the topic's importance, a wide variety of empirical methods have been applied to estimate the form that yield distributions take. Most published results have found some degree of skewness in yields, but progress on providing a rationale for the presence of skewness has been very limited.

Emphasizing microfoundations, this article has used the Law of the Minimum together with structure on the input-conditioned resource availabilities to provide a better understanding of the stochastic attributes of crop yield distributions at the small-plot level of analysis. Yield skewness was considered for three types of resource availability distributions. It was shown that the LoM can support both positive and negative yield skewness. Location and scale heterogeneities were studied to discern definite, but sometimes involved 
patterns in their implications for skewness. It was suggested that the left-tail attributes of resource availability distributions are key in determining yield skew.

One finding is that negative skewness will arise when the left tails of resource availabilities are thin and there is heterogeneity in means of resource availabilities, as may arise when one input (say nitrogen) becomes cheap. For rain-fed cropland, the twentieth century saw great strides in managing nutrient availability through increased use of synthetic fertilizers (Federico 2005). But weather susceptibility has remained more problematic. Another finding is that when some resource availabilities in the crop production process are quite tightly controlled, then the left tails of resource availabilities should be thin and negative skewness will be favored. This suggests that one should be more likely to compute negative skewness when looking at yield data of more recent vintage in prime growing areas and in more developed countries where market inputs are more readily available. This is consistent with our reading of findings for the United States Corn Belt, as reviewed in the introduction. On the other hand some technologies (such as irrigation) may completely remove the left tail of a yield distribution. So there are also reasons to believe that yield skewness should become more positive over time in some parts of the world.

It may appear premature to inquire into why skewness could change across crops, geography, technological availabilities, and time when there remains incomplete consensus on the sign of skewness. But we believe otherwise. To some extent, disagreement on the sign may be because skewness is not uniformly signed across crops, agronomic conditions, available technologies, and market prices. Hypotheses on the origin of skewness may rationalize the different estimates so that more discerning statistical tests can be run and, ultimately, so that modeling of yield distributions can be conditioned on the most relevant features of the crop's production environment.

\section{[Received June 2007; accepted April 2008.]}

\section{References}

Atwood, J., S. Shaik, and M. Watts. 2002. "Can Normality of Yields be Assumed for Crop Insurance?" Canadian Journal of Agricultural Economics 50:171-84.
—. 2003. "Are Crop Yields Normally Distributed? A Reexamination." American Journal of Agricultural Economics 85:888-901.

Berck, P., J. Geoghegan, and S. Stohs. 2000. "A Strong Test of the von Liebig Hypothesis." American Journal of Agricultural Economics 82(November):948-955.

Berck, P., and G. Helfand. 1990. "Reconciling the von Liebig and Differentiable Crop Production Functions." American Journal of Agricultural Economics 72:985-96.

Bloom, A.J., F.S. Chapin, III, and H.A. Mooney. 1985. "Resource Limitations in Plants-An Economic Analogy." Annual Review of Ecology and Systematics 16:363-92.

Bose, R.C., and S.S. Gupta. 1959. "Moments of Order Statistics from a Normal Population." Biometrika 46:433-440.

Boyle, P.P., M. Broadie, and P. Glasserman. 1997. "Monte Carlo Methods for Security Pricing." Journal of Economic Dynamics and Control 21:1267-321.

Cerrato, M.E., and A.M. Blackmer. 1990. "Comparison of Models for Describing Corn Yield Response to Nitrogen Fertilizer." Agronomy Journal 82:138-43.

Chambers, R.G., and E. Lichtenberg. 1996. "A Nonparametric Approach to the von Liebig-Paris Technology." American Journal of Agricultural Economics 78:373-86.

Choi, J.-K. 2005. "Letter to the Editor." Cотmunications in Statistics-Theory and Methods 34(4):997-8.

Cornes, R. 1993. "Dike Maintenance and Other Stories: Some Neglected Types of Public Goods." Quarterly Journal of Economics 108:259-71.

Dasgupta, P., and E. Maskin. 1987. "The Simple Economics of Research Portfolios." Economic Journal 97:581-95.

David, H.A., and H.N. Nagaraja. 2003. Order Statistics. Hoboken, NJ: Wiley.

Day, R.H. 1965. "Probability Distributions of Field Crop Yields." Journal of Farm Economics 47:713-41.

Dow, W.H., T.J. Philipson, and X. Sala-I-Martin. 1999. "Longevity Complementarities Under Competing Risks." American Economic Review 89:1358-71.

Durrett, R. 1996. Probability: Theory and Examples, 2nd ed. Belmont, CA: Duxbury Press.

Eugene, N., C. Lee, and F. Famoye. 2002. "Beta-Normal Distribution and Its Applications." Communications in Statistics-Theory and Methods 31(4):497-512.

Federico, G. 2005. Feeding the World: An Economic History of Agriculture, 1800-2000. Princeton NJ: Princeton University Press. 
Frank, M.D., B.R. Beattie, and M.E. Embleton. 1990. "A Comparison of Alternative Crop Response Models." American Journal of Agricultural Economics 72:597-603.

Gallagher, P. 1987. "U.S. Soybean Yields: Estimation and Forecasting with Nonsymmetric Disturbances." American Journal of Agricultural Economics 69:796-803.

Gupta, A.K., and S. Nadarajah. 2004a. "Order Statistics and Records." In A.K. Gupta and S. Nadarajah, eds. Handbook of Beta Distribution and Its Applications, pp. 88-96. New York: Marcel Dekker.

- 2004b. "On the Moments of the Beta-Normal Distribution." Communications in StatisticsTheory and Methods 33(1):1-13.

Hennessy, D.A. 2008. "AJAE Appendix: Crop Yield Skewness Under Law of the Minimum Technology." Unpublished manuscript. Available at: http://agecon.lib.umn.edu/.

Hirshleifer, J. 1983. "From Weakest-Link to BestShot: The Voluntary Provision of Public Goods." Public Choice 41:371-386.

Joernsgaard, B., and S. Halmoe. 2003. "Intra-field Yield Variation over Crops and Years." European Journal of Agronomy 19:23-33.

Jones, M.C. 2004. "Letter to the Editor: The Moments of the Beta-Normal Distribution with Integer Parameters are the Moments of Order Statistics from the Normal Distribution." Communications in Statistics-Theory and Methods 33(11):2869-70.

Just, R.E., and Q. Weninger. 1999. “Are Crop Yields Normally Distributed?" American Journal of Agricultural Economics 81:287-304.
Ker, A.P., and B.K. Goodwin. 2000. "Nonparametric Estimation of Crop Insurance Rates Revisited." American Journal of Agricultural Economics 83:463-78.

Moss, C.B., and J.S. Shonkwiler. 1993. "Estimating Yield Distributions with a Stochastic Trend and Nonnormal Errors." American Journal of Agricultural Economics 75:1056-62.

Nelson, C.H. 1990. "The Influence of Distributional Assumptions on the Calculation of Crop Insurance Premia." North Central Journal of Agricultural Economics 12:71-8.

Nelson, C.H., and P.V. Preckel. 1989. "The Conditional Beta Distribution as a Stochastic Production Function." American Journal of Agricultural Economics 71:370-78.

Paris, Q. 1992. "The von Liebig Hypothesis." American Journal of Agricultural Economics 74:1019-28.

Ramirez, O.A., S. Misra, and J. Field. 2003. "Crop Yield Distributions Revisited." American Journal of Agricultural Economics 85:108-20.

Rubio, G., J. Zhu, and J.P. Lynch. 2003. “A Critical Test of the Two Prevailing Theories of Plant Response to Nutrient Availability." American Journal of Botany 90:143-52.

Sherrick, B.J., F.C. Zanini, G.D. Schnitkey, and S.H. Irwin. 2004. "Crop Insurance Valuation Under Alternative Yield Distributions." American Journal of Agricultural Economics 71:406-19.

Swinton, S.M., and R.P. King. 1991. "Evaluating Robust Regression Techniques for Detrending Crop Yield Data with Nonnormal Errors." American Journal of Agricultural Economics 73:446-51. 
lowa State University does not discriminate on the basis of race, color, age, religion, national origin, sexual orientation, gender identity, genetic information, sex, marital status, disability, or status as a U.S. veteran. Inquiries can be directed to the Director of Equal Opportunity and Compliance, 3280 Beardshear Hall, (515) 294-7612. 